\title{
Donor-Specific Transcriptomic Analysis of Alzheimer's Disease-Associated Hypometabolism Highlights a Unique Donor, Ribosomal Proteins and Microglia
}

\author{
Sejal Patel, ${ }^{1}$ Derek Howard, ${ }^{1}$ Alana Man, ${ }^{1,2}$ Deborah Schwartz, ${ }^{3,4}$ Joelle Jee, ${ }^{1,5}$ Daniel Felsky, ${ }^{1}$ \\ Zdenka Pausova, ${ }^{6}$ Tomas Paus, ${ }^{4,7,8}$ and ${ }^{-}$Leon French ${ }^{1,7,8,9}$
}

\section{https://doi.org/10.1523/ENEURO.0255-20.2020}

${ }^{1}$ Krembil Centre for Neuroinformatics, Centre for Addiction and Mental Health, Toronto, Ontario M5T 1L8, Canada, ${ }^{2}$ Victoria College, University of Toronto, Toronto, Ontario M5S 1K7, Canada, ${ }^{3}$ Rotman Research Institute, Baycrest Centre for Geriatric Care, University of Toronto, Toronto, Ontario M6A 2E1, Canada, ${ }^{4}$ Department of Psychology, University of Toronto, Toronto, Ontario M5S 3G3, Canada, ${ }^{5}$ Faculty of Arts and Science, University of Toronto, Toronto, Ontario M5S 3G3, Canada, ${ }^{6}$ The Hospital for Sick Children, University of Toronto, Toronto, Ontario M5G 1X8, Canada, ${ }^{7}$ Department of Psychiatry, University of Toronto, Ontario M5S 3G3, Toronto, ${ }^{8}$ Institute for Medical Science, University of Toronto, Toronto, Ontario M5S 1A8, Canada, and ${ }^{9}$ Campbell Family Mental Health Research Institute, Centre for Addiction and Mental Health, Toronto, Ontario M5T 1L8, Canada

\section{Visual Abstract}

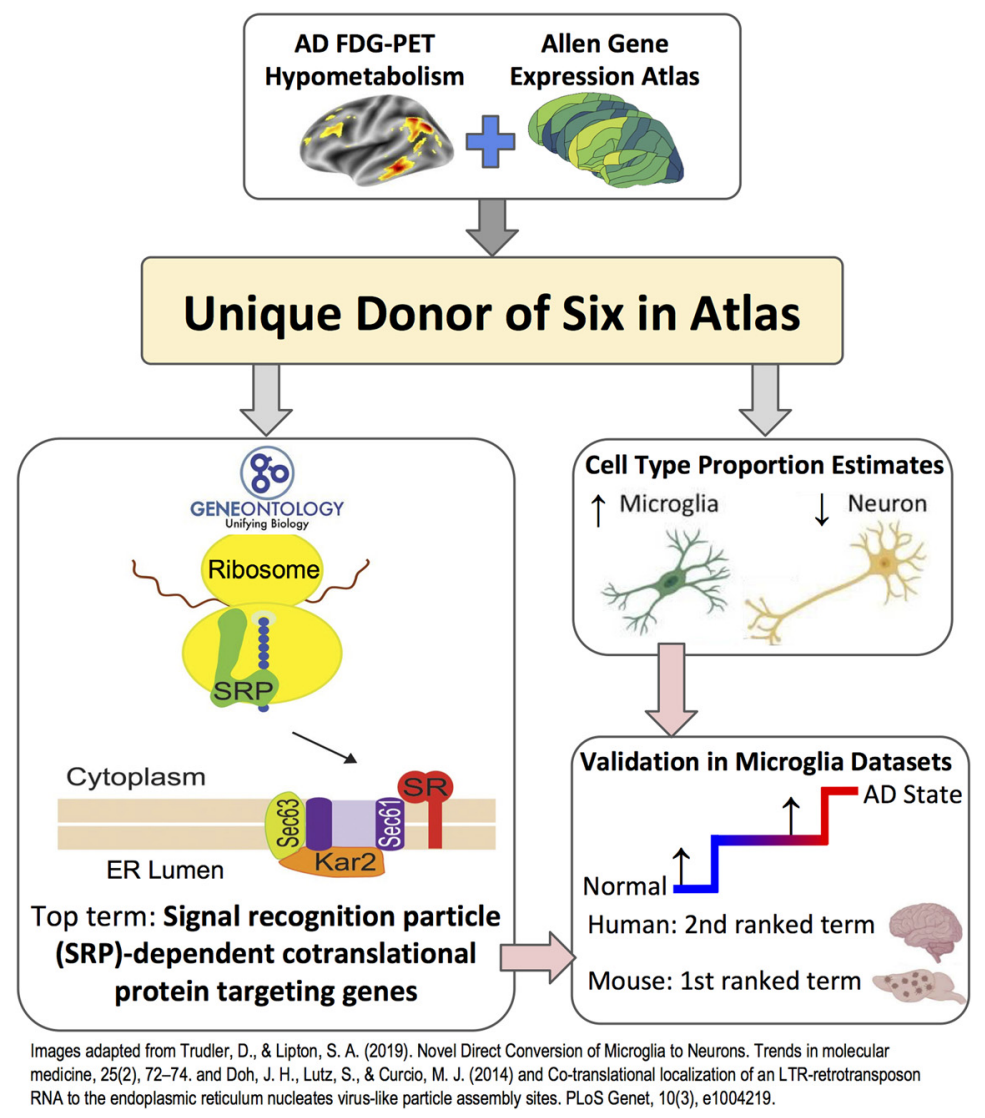

Alzheimer's disease (AD) starts decades before clinical symptoms appear. Low-glucose utilization in regions of the cerebral cortex marks early AD. To identify these regions, we conducted a voxel-wise meta-analysis of 


\section{Significance Statement}

Fluorodeoxyglucose positron emission tomography (FDG-PET) is a frontline tool for the diagnosis of dementia. We sought to determine the molecular underpinnings of the metabolic signatures of Alzheimer's disease (AD) revealed by FDG-PET. We found that of the six brains in the Allen Human Brain Atlas, a set of ribosomal proteins strongly aligned with the hypometabolism map in one of the six Atlased brains. While this brain was from a 39-year-old, it contained a neurofibrillary tangle in the entorhinal cortex. We observe changes in estimated neuron and microglia proportions that also suggest this individual had prodromal AD. In other studies, expression of the ribosomal genes increases across AD-associated microglial activation.

previous studies conducted with positron emission tomography that compared AD patients with healthy controls. The resulting map marks hypometabolism in the posterior cingulate, middle frontal, angular gyrus, and middle and inferior temporal regions. Using the Allen Human Brain Atlas, we identified genes that show spatial correlation across the cerebral cortex between their expression and this hypometabolism. Of the six brains in the Atlas, one demonstrated a strong spatial correlation between gene expression and hypometabolism. Previous neuropathological assessment of this brain from a 39-year-old male noted a neurofibrillary tangle in the entorhinal cortex. Using the transcriptomic data, we estimate lower proportions of neurons and more microglia in the hypometabolic regions when comparing this donor's brain with the other five donors. Within this single brain, signal recognition particle (SRP)-dependent cotranslational protein targeting genes, which encode primarily cytosolic ribosome proteins, are highly expressed in the hypometabolic regions. Analyses of human and mouse data show that expression of these genes increases progressively across AD-associated states of microglial activation. In addition, genes involved in cell killing, chronic inflammation, ubiquitination, tRNA aminoacylation, and vacuole sorting are associated with the hypometabolism map. These genes suggest disruption of the protein life cycle and neuroimmune activation. Taken together, our molecular characterization reveals a link to $A D$-associated hypometabolism that may be relevant to preclinical stages of $A D$.

Key words: microglia; neurodegeneration; neuroinflammation; transcriptomics

\section{Introduction}

Alzheimer's disease $(A D)$, one of the most prevalent neurodegenerative diseases, is thought to affect $\sim 5 \%$ of those aged 60 years and above worldwide (Qiu et al., 2009). It is the most common form of dementia, which is clinically characterized by a severe decline in cognitive

Received June 12, 2020; accepted October 19, 2020; First published November 19, 2020.

L.F. owns shares in Cortexyme Inc., a company that is developing a gingipain inhibitor to treat Alzheimer's Disease. All other authors declare no competing financial interests.

Author contributions: S.P., D.H., D.S., Z.P., T.P., and L.F. designed research; S.P., D.H., A.M., D.S., J.J., Z.P., T.P., and L.F. performed research; S.P., D.H., A.M., D.S., J.J., Z.P., T.P., and L.F. contributed unpublished reagents/analytic tools; S.P., D.H., A.M., D.S., J.J., D.F., Z.P., T.P., and L.F. analyzed data; S.P., D.H., D.F., T.P., and L.F. wrote the paper.

This work was supported by the CAMH Foundation, CAMH Discovery Fund, and a National Science and Engineering Research Council of Canada (NSERC) Discovery Grant (L.F.).

Acknowledgements: We thank Taylor Schmitz and Spiro Pantazatos for analyzing MRI scans. We also thank the Allen Institute for Brain Science for creating the transcriptomic atlas of the human brain and Ed Lein, Michael Hawrylycz, Jeremy Miller, Taylor Schmitz, and Shreejoy Tripathy for their insightful comments and suggestions.

Correspondence should be addressed to Leon French at leonfrench@ gmail.com.

https://doi.org/10.1523/ENEURO.0255-20.2020

Copyright @ 2020 Patel et al.

This is an open-access article distributed under the terms of the Creative Commons Attribution 4.0 International license, which permits unrestricted use, distribution and reproduction in any medium provided that the original work is properly attributed. functioning and defined neuropathologically by the emergence and topographical progression of amyloid plaques, neurofibrillary tangles, and neuronal loss (Masters et al., 2015).

Currently, fluorodeoxyglucose positron emission tomography (FDG-PET) is a primary frontline tool for diagnosing dementia and its subtypes. FDG-PET uses a radioactive tracer, [18F] FDG-PET, to measure glucose metabolism within the brain (Friedland et al., 1983), with altered cerebral glucose metabolism indicating $A D$ with high sensitivity and specificity (Mosconi et al., 2008). Importantly, hypometabolism patterns can be seen in atrisk individuals decades before the development of symptoms (Reiman et al., 2004; Mosconi et al., 2006; Langbaum et al., 2010; Landau et al., 2011; Chen et al., 2012). This timing supports the concept that $A D$ exists on a spectrum or continuum of pathologies that includes stages of subtle cognitive decline, mild cognitive impairment, and dementia (Albert et al., 2011; McKhann et al., 2011). Despite the clear link between metabolic changes measured by FDG-PET and risk for AD, it remains unclear which etiopathological mechanisms are responsible for driving these changes.

Using the Allen Human Brain Atlas, we sought to characterize the pattern of regional hypometabolism found in patients with $A D$. By integrating this atlas with a meta-analytic map of FDG-PET differences, we identified genes with spatial expression patterns similar to that of the lower glucose metabolism in the AD brain. This transcriptomic approach was performed to identify consistent 
molecular markers of the FDG-PET pattern. To test the consistency of these markers, we performed the transcriptomic analysis within each of the six donors, which revealed a surprisingly strong association in a single donor. To better understand this signal, we examined cell-type proportion estimates. To validate this molecular and cell-type-specific marker of the FDG-PET pattern, we examined the relevant genes and cell-type in two datasets that profiled gene expression across AD-associated states.

\section{Materials and Methods}

\section{Meta-analysis of Alzheimer's FDG-PET studies}

We performed a meta-analysis of FDG-PET studies that compared, at rest, Alzheimer's patients with healthy controls. To compile a list of studies, a literature search was conducted on studies from January 1985 to January 2012. We used the following search query: [FDG-PET OR positron emission tomography $\mathrm{OR}$ fluorodeoxyglucose OR glucose metabolism] AND [dementia]. Studies were examined to fulfill the following criteria: (1) original research papers available in English (no case studies or reviews); (2) participants examined using [18F] FDG-PET at rest (no functional tasks); (3) AD patients compared with age-matched healthy controls; (4) clinical diagnosis of $A D$ using NINCDS-ADRDA (McKhann et al., 1984) or DSM-IV (American Psychiatric Association, 1994) criteria; and (5) whole-brain analyses (no region-of-interest analyses) conducted in standardized stereotaxic space with available coordinates. Each article was read twice to determine whether the study met the inclusion criteria.

Coordinates of regional hypometabolism peaks from retained articles were used to create ALE maps using BrainMap's GingerALE application (www.brainmap.org/ ale; Eickhoff et al., 2009). This software assigns each voxel an activation likelihood estimate equal to the probability of at least one of the reported peaks of hypometabolism being located in that voxel (Turkeltaub et al., 2002). These voxelwise maps were clustered to find distinct anatomic clusters [min cluster extent $=500 \mathrm{~mm}^{3}$; false discovery rate (FDR) $q=0.05]$. The identified clusters were then used to determine a threshold that marks which samples are inside regions of hypometabolism.

\section{Gene expression data}

We used the Allen Human Brain Atlas to identify genes with spatial expression patterns similar to the FDG-PET hypometabolism map. This Atlas provides a comprehensive transcriptional landscape of the adult human brain (Hawrylycz et al., 2012). The Atlas was obtained from six individuals (five males, one female), with age ranging from 24 to 57 years. Custom 64K Agilent microarrays were used to assay genome-wide expression in 3702 spatiallyresolved samples (232 named brain regions). We also used the RNA-sequencing datasets that were generated on the Illumina HiSeq2000 platform. These RNA-sequencing data were quantified with transcripts per million (TPM) and assayed a subset of anatomic structures from two of the six brains. The Allen Institute normalized the data and adjusted for array-specific biases, batch, and dissection method. Microarray probes were filtered using quality control data provided by Miller et al. (2014). After this filtering, 31,452 probes remained of the 58,692 on the microarray.

\section{Differential expression analyses}

The Allen Human Brain Atlas gene expression data were first used at the sample and donor level to identify genes that are differentially expressed in the regions of hypometabolism identified by the ALE-based analysis. Expression values were mean-averaged for genes with multiple probes, resulting in 15,143 genes. This analysis was restricted to samples from the cerebral cortex, as marked by the Allen Human Brain Atlas annotations (allocortical regions, namely the hippocampal formation and piriform cortex, were excluded). For each donor and gene, expression values were compared between samples inside and outside of the hypometabolic regions using the Mann-Whitney $U$ test. The Allen Institute provided MNI coordinates, which were used to map expression values into the voxel space of the meta-analysis. For analyses that spanned multiple donors, Fisher's method was used to generate a single meta $p$ value for each gene and direction (Fisher, 1925). We used the BenjaminiHochberg FDR procedure for multiple test correction to adjust for the many tested genes (Benjamini and Hochberg, 1995).

\section{Gene Ontology (GO) enrichment analysis}

The GO provides gene-level annotations that span specific cellular components, biological processes, and molecular functions (Ashburner et al., 2000). These annotations, defined by GO terms, were required to have annotations for 10-200 tested genes (6333 GO groups annotating 14,241 unique genes). To test for enrichment, we sorted the genes from the most overexpressed to underexpressed in regions of hypometabolism. Within this ranking, the area under the receiver operating characteristic curve (AUC) was used to test for GO terms that are enriched in either direction (overexpressed: $A \cup C>0.5$, underexpressed: AUC $<0.5$ ). The Mann-Whitney $U$ test was used to determine statistical significance with FDR correction for the GO groups tested. We used GO annotations from the GO.db and org.Hs.eg.db packages in R, version 3.8.2, which were dated April 24, 2019 (Carlson, 2016a,b). We used the REVIGO tool to summarize many terms that were significant after correction (Supek et al., 2011). We used the default REVIGO parameters with uncorrected $p$ values for the input GO groups and restricted this analysis to the biological process branch of the GO.

\section{Estimation of cell-type proportions}

The Marker Gene Profile (MGP) tool was used to estimate cell-type proportions from the cerebral cortex expression profiles (Mancarci et al., 2017). This method uses the first principal component of the expression of cell-type-specific genes to estimate the relative abundance of a cell type. We used 21 top marker genes from a 
single cell study of the adult human temporal cortex (Darmanis et al., 2015; their Supplementary Table S3). This study used transcriptomic profiles to cluster cells into astrocyte, neuron, oligodendrocyte, oligodendrocyte precursor, microglia, and endothelial groups. These marker genes were used to calculate AUC values and estimate cell-type proportions with the MGP tool. Proportions were estimated separately for each donor across the same cortical samples used in the differential expression analysis.

\section{Single-cell RNA sequencing analysis of mouse microglia}

Supplemental data from a single-cell RNA sequencing study of wild-type and $A D$ transgenic mouse model (5XFAD) were used to examine gene expression in immune cell types (Keren-Shaul et al., 2017). Keren-Shaul and colleagues profiled trancriptomically 8016 immune cells from three wild-type and three 5XFAD mice and clustered these cells into 10 distinct subpopulations based on expression. Of these 10 clusters, three expressed microglia markers. Two of these microglia clusters contained cells primarily from 5XFAD and not wild-type mice and named them disease-associated microglia (DAM). For our analysis, we consider these clusters separately as different microglial states: normal, intermediate (Group II DAM), and $A D$ associated (Group III DAM).

\section{Single-nucleus RNA sequencing analysis}

Supplemental data from a single-nucleus RNA sequencing study of the human prefrontal cortex were used to examine differential expression across AD states in microglia. Specifically, for each gene, we extracted adjusted $p$ values (IndModel.adj.pvals), mean expression, and fold changes (IndModel.FC) from Mathys et al. (2019; their Supplement Table 2). After quality control, Mathys et al. (2019) clustered the transcriptomes of 70,634 nuclei from 48 individuals into eight broad cell-type clusters. For this work, we focused on data from the 1920 microglia nuclei. The 48 participants in this study were classified into no (24), early (15), and late (9) AD pathology. To test for enrichment of our genes of interest, we sorted the genes from the most overexpressed to underexpressed for the differential expression results for no versus early pathology and early versus late pathology analyses. Within this ranking, the AUC was used to test for significantly enriched genes in either direction. We also used the mean expression to determine which genes increase in expression across the three pathology groups. For a given set of genes, the hypergeometric test was used to determine whether a greater number of genes increase across pathology than expected by chance.

\section{Code accessibility}

Scripts for reproducing the analyses are publicly available online at https://github.com/leonfrench/ADAllen-FDG and https://figshare.com/articles/dataset/ Donor_specific_transcriptomic_analysis_of_Alzheimer_s_ disease_associated_hypometabolism_highlights_a_unique donor_microglia_and_ribosomal_prot eins/12233552 and as Extended Data 1.

\section{Results}

\section{Meta-analysis of FDG-PET studies of AD}

Our literature search for FDG-PET studies identified 3229 titles. Screening of the abstracts yielded 230 relevant studies. Upon review of the full articles, 29 relevant studies remained. When two studies used the same patient population, one of the overlapping studies was excluded, resulting in a total of 27 studies yielding 33 independent samples with a total of 915 Alzheimer's patients and 715 healthy controls (details in Extended Data Fig. 1-1). Activation likelihood estimation (ALE) meta-analysis of these studies identified the following cortical regions as showing (consistently) lower glucose metabolism in patients versus controls: posterior cingulate gyrus, middle frontal region, angular gyrus, and middle and inferior temporal regions. A cluster analysis revealed 23 clusters (min cluster extent $=500 \mathrm{~mm}^{3}$; FDR $q=0.05$ ). A voxelwise threshold of 0.006 was set to mirror this clustering map (Fig. 1) and was used to determine whether a given voxel was inside an AD-associated region of hypometabolism in subsequent transcriptomic analyses.

\section{Many genes are differentially expressed in cortical regions with $A D$-associated hypometabolism}

To identify molecular signatures underlying $A D$ hypometabolism, we next performed a transcriptome-wide analysis to test for genes that correlate with the FDG-PET derived map. Using all six brains included in the Allen Atlas, we first identified the genes that were differentially expressed in the FDG-PET-defined hypometabolic regions of the cerebral cortex (one female and five males, aged 24-57 years). The number of cerebral cortex samples profiled by the Allen Institute ranged from 182 to 481 per donor; $5.9-9.9 \%$ overlapped with the hypometabolic regions. Of the 15,143 genes tested, 99 were significantly expressed at higher levels, and 51 at lower levels in these hypometabolic regions, after correction, across all donors. Substantial variability across the six brains in the Allen Human Brain Atlas has been previously noted both genome-wide and in the context of $A D$ (French and Paus, 2015; Hawrylycz et al., 2015; Grothe et al., 2018; Ritchie et al., 2018). Given this variability, we then tested each brain separately. Strikingly, one brain drove the majority of the above atlas-wide signal for spatial expression overlap with the FDG-PET-derived map. In this brain (10021/ H0351.2002), 647 genes were differentially expressed, with $74 \%$ being expressed at lower levels in the hypometabolic regions. In the remaining five donor brains, differentially expressed genes were only found in the oldest donor (donor 12876/H0351.1009, 57-year-old male). Taken together, our analysis of brain 10021/H0351.2002 marks it as an outlier with hundreds of genes that align spatially with the patterns of lower glucose metabolism observed in patients with $A D$ (vs controls).

\section{Brain-specific analyses point to a unique donor}

We examined the demographic information and metadata of this donor to help understand the above observation. Brain 10021/H0351.2002 was from a 39-year-old 

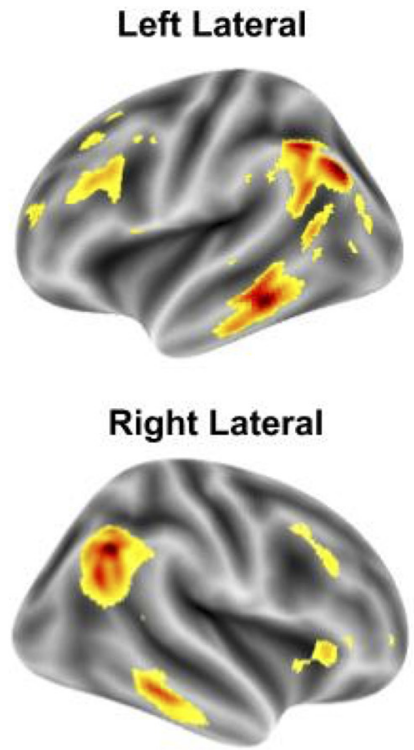
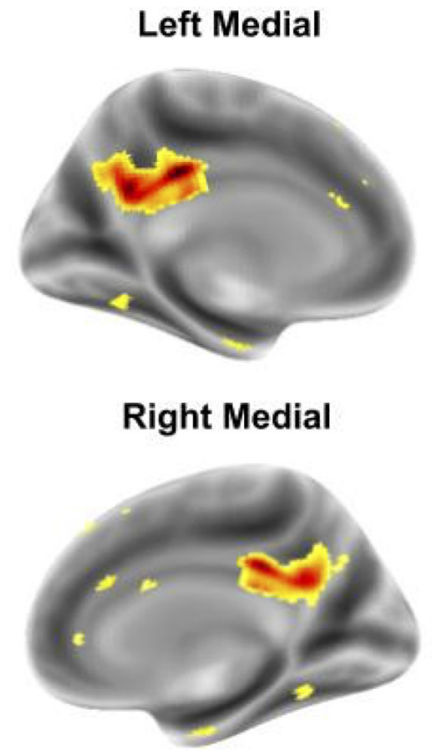

ALE Value

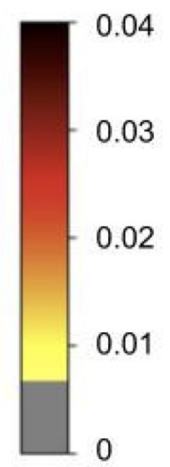

Figure 1. Cortical surface views of the ALE meta-analysis results. Regions where hypometabolism was not detected are transparent (ALE value of 0.006 or less). Lower glucose utilization (AD vs controls) ranges from low (yellow) to high (black).

male African American individual. The postmortem interval was $10 \mathrm{~h}$, the lowest of the six donors. In agreement, RNA integrity values (RINs) for this brain are higher than the other donors for all four regions assayed for RIN (frontal pole: 7.5, occipital pole: 7.1, cerebellum: 8.6, and brainstem: 7.3). As documented by the Allen Institute, this donor, like the others, had no known history of neuropsychiatric or neurologic conditions. The presence of a broad range of drugs was tested for in postmortem blood by the Allen Institute. In donor $10021 / \mathrm{H} 0351.2002$, atropine, caffeine, lidocaine, and monoethylglycinexylidide were detected at levels usually not toxicologically significant. We note that monoethylglycinexylidide is a metabolite of lidocaine, an anesthetic and antiarrhythmic agent. Among the six donors, only 10021/H0351.2002 tested positive for lidocaine and monoethylglycinexylidide. The included brains were also classified as "normal" by a radiologist or pathologist. While considered neurotypical, it was noted that $10021 / \mathrm{H} 0351.2002$ contained a single neurofibrillary tangle in the entorhinal cortex. Neurofibrillary tangles in the hippocampus and entorhinal cortex are considered early events in AD progression (Guillozet et al., 2003). Neurofibrillary tangles were not found in the other five brains (three of which are older than this donor). The presence of a neurofibrillary tangle is a unique feature of this individual. The postmortem interval and RIN values suggest that tissue quality is not driving the Alzheimer's-associated molecular patterns observed.

\section{ER translocation genes are enriched for overexpression in areas of Alzheimer's-associated hypometabolism}

To distil the molecular results, we performed GO enrichment analysis on the transcriptome-wide results from donor brain 10021/H0351.2002. In total, $215 \mathrm{GO}$ groups were significantly enriched. Table 1 shows the top $10 \mathrm{GO}$ terms enriched for genes upregulated in hypometabolic regions and Extended Data Table 1-1 contains complete enrichment results for all donors separately. Because of the high degree of overlap in gene membership among our top GO terms, we used REVIGO tool to summarize them (Supek et al., 2011). This tool removes redundant GO terms based on semantic similarity, providing a dispensability metric. Of the 98 biological process terms enriched for overexpression, three were assigned the lowest possible dispensability score of zero: SRP-dependent cotranslational protein targeting to membrane (GO:0006614, 87 genes, $\mathrm{AUC}=0.874, p_{\mathrm{FDR}}<$ $10^{-28}$ ), chronic inflammatory response (GO:0002544, 15 genes, $\left.A \cup C=0.78, p_{\text {FDR }}<0.05\right)$, and cell killing (GO:0001906, 94 genes, AUC $=0.60, p_{\text {FDR }}<0.05$ ). The strongest signal is from genes involved in SRP-dependent cotranslational protein targeting to membrane (Fig. 2). This process targets protein translocation to the endoplasmic reticulum via the signal-recognition particle (SRP). These genes are primarily components of the cytosolic ribosome and henceforth referred to as "ER translocation" genes. Six of these genes are found within the top 20 genes with higher expression in hypometabolic regions ( $R P L 34, R P L 32, R P S 27, R P S 27 A$, $R P L 37 A$, and RPS15A). In contrast, genes that are underexpressed in regions of hypometabolism are less significantly enriched for specific GO terms (lowest $\left.p_{\text {FDR }}=7.3 \times 10^{-8}\right)$. However, these top terms contain more diverse themes (Table 1, bottom half), some of which have been previously implicated in AD. The most significant GO terms representing these themes are: "ubiquitin ligase complex", "tRNA aminoacylation," "ATPase activity, coupled," "HOPS complex" (involved in endosomal vesicle tethering), and "microtubule organizing center part." The ubiquitin-proteasome system has been linked to $A D$ (Oddo, 2008). Of the four genes that encode ubiquitin, three with available data are strongly overexpressed in 
Table 1: Top GO groups enriched for differential expression in areas of AD-associated hypometabolism in brain 10 021/ H0351.2002

\begin{tabular}{|c|c|c|c|c|}
\hline Name & Genes & ID & AUC & $p$ value $_{\mathrm{FDR}}$ \\
\hline SRP-dependent cotranslational protein targeting to membrane & 87 & GO:0006614 & 0.874 & $1.35 \mathrm{E}-29$ \\
\hline Cotranslational protein targeting to membrane & 90 & GO:0006613 & 0.865 & 2.07E-29 \\
\hline Protein targeting to ER & 92 & GO:0045047 & 0.847 & $2.86 \mathrm{E}-27$ \\
\hline Cytosolic ribosome & 87 & GO:0022626 & 0.856 & $3.45 \mathrm{E}-27$ \\
\hline Establishment of protein localization to endoplasmic reticulum & 96 & GO:0072599 & 0.828 & $1.66 \mathrm{E}-25$ \\
\hline Structural constituent of ribosome & 107 & GO:0003735 & 0.794 & $1.05 E-22$ \\
\hline Ribosomal subunit & 158 & GO:0044391 & 0.737 & $1.01 \mathrm{E}-21$ \\
\hline Nuclear-transcribed mRNA catabolic process, nonsense-mediated decay & 104 & GO:0000184 & 0.783 & 2.07E-20 \\
\hline Protein localization to endoplasmic reticulum & 109 & GO:0070972 & 0.765 & $9.44 \mathrm{E}-19$ \\
\hline Cytosolic large ribosomal subunit & 47 & GO:0022625 & 0.894 & $6.73 E-18$ \\
\hline microtubule organizing center part & 145 & GO:0044450 & 0.395 & 0.00244 \\
\hline DNA-dependent ATPase activity & 59 & GO:0008094 & 0.33 & 0.00145 \\
\hline HOPS complex & 13 & GO:0030897 & 0.137 & 0.00135 \\
\hline ATPase activity, coupled & 186 & GO:0042623 & 0.396 & 0.00026 \\
\hline tRNA aminoacylation for protein translation & 40 & GO:0006418 & 0.268 & 9.84E-05 \\
\hline Amino acid activation & 43 & GO:0043038 & 0.275 & 8.24E-05 \\
\hline Aminoacyl-tRNA ligase activity & 33 & GO:0004812 & 0.243 & $8.24 \mathrm{E}-05$ \\
\hline Cullin-RING ubiquitin ligase complex & 111 & GO:0031461 & 0.355 & 3.84E-05 \\
\hline tRNA aminoacylation & 42 & GO:0043039 & 0.259 & $1.91 \mathrm{E}-05$ \\
\hline Ubiquitin ligase complex & 195 & GO:0000151 & 0.368 & 7.35E-08 \\
\hline
\end{tabular}

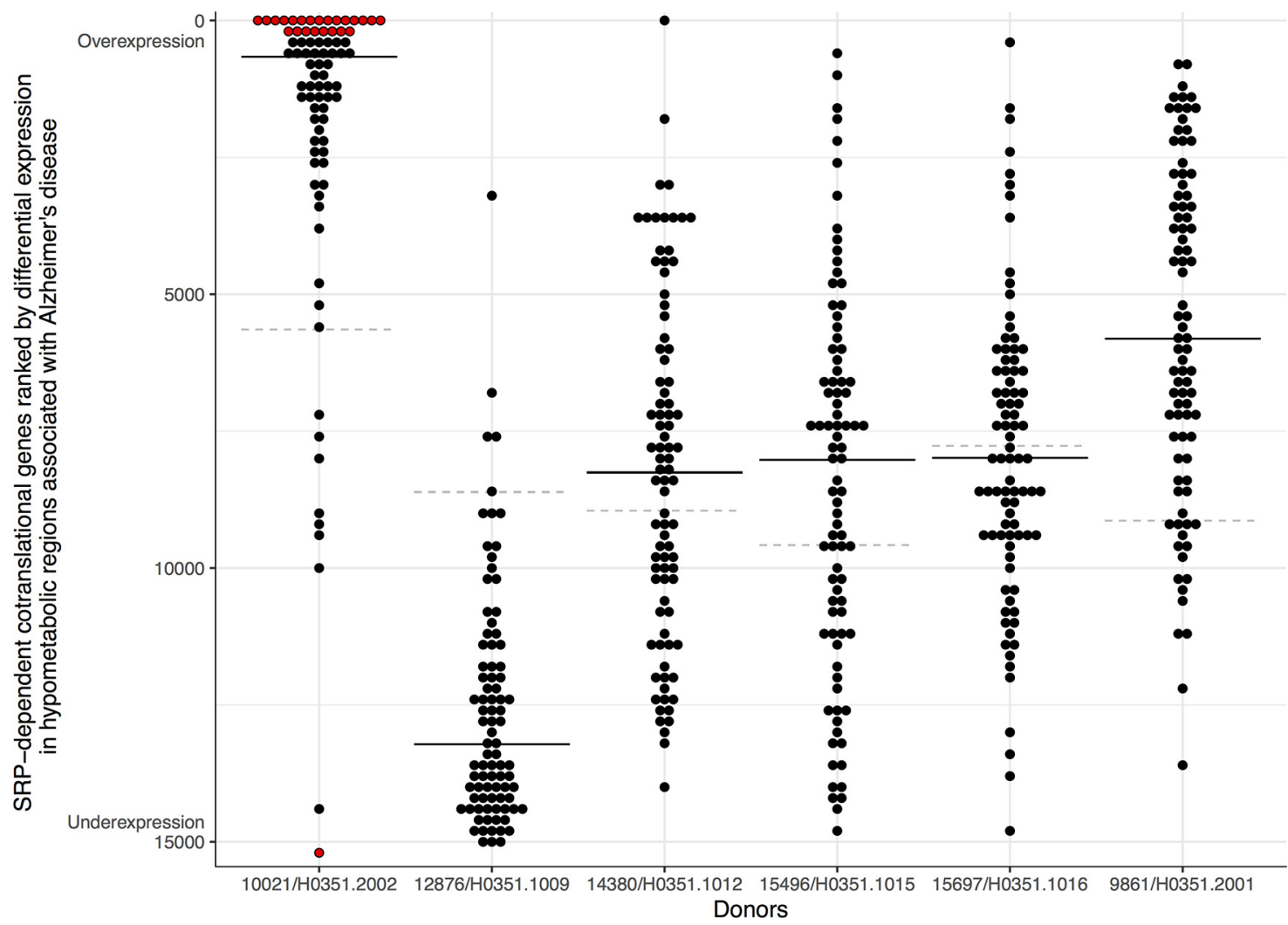

Figure 2. SRP-dependent cotranslational genes ranked based on differential expression in hypometabolic regions associated with AD. Genes are marked with dots, with the $y$-axis representing the genome-wide differential expression rank and ranges from overexpression (top) to underexpression (bottom). The black line marks the median expression rank of the SRP-dependent cotranslational genes. The dashed gray line marks the gene with the most stable expression between inside and outside of each donor's hypometabolic regions. Red highlights genes that pass correction for multiple testing. 


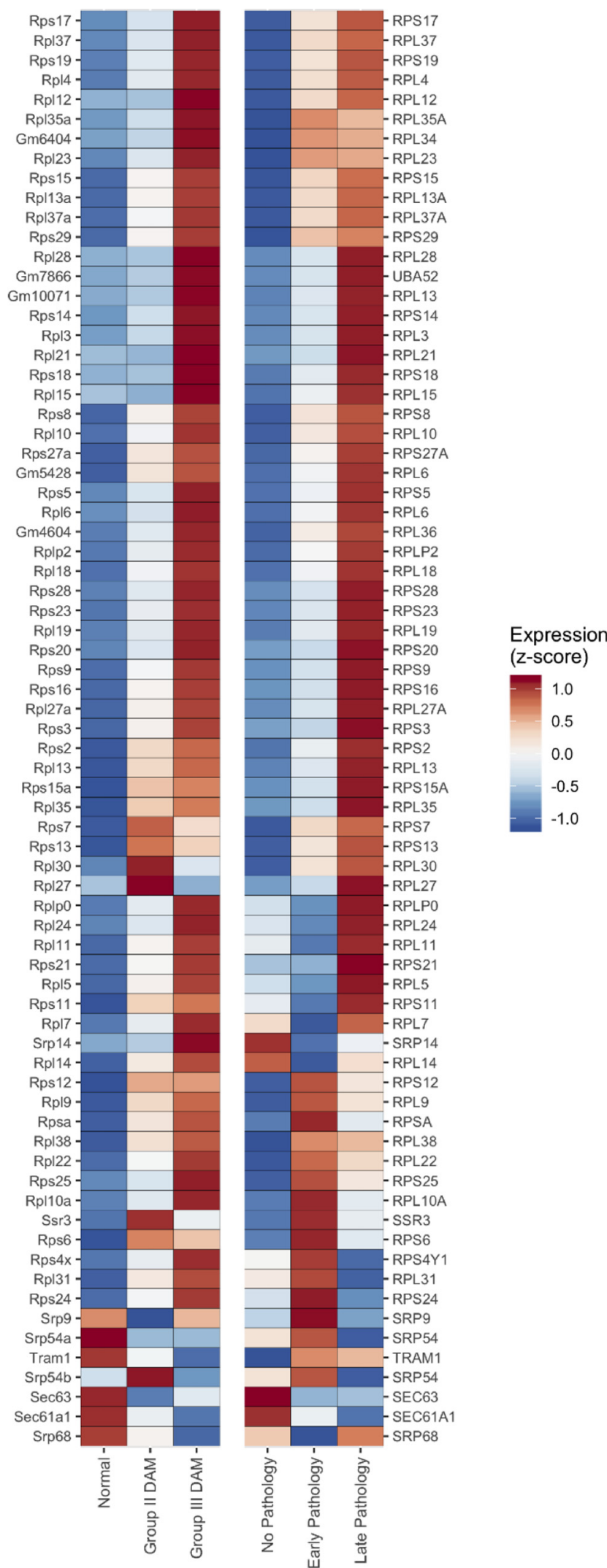

Figure 3. Heatmap of the ER translocation gene expression across three microglia cell clusters from the AD mouse model (left half) and $A D$ pathology subgroups (right half). Expression for each gene is $z$-scored with high expression in red and low in regions of hypometabolism in this brain. In summary, this enrichment analysis points to spatial differences in vesicle fusion, protein translation, targeting, and degradation.

\section{Validation of ER translocation gene enrichment with RNA sequencing data}

Focusing on donor $10021 / \mathrm{H} 0351.2002$, the top-ranked GO group, "SRP-dependent cotranslational protein targeting to membrane"/“ER translocation," contains genes that are involved in the targeting of proteins to the endoplasmic reticulum. Given the high and ubiquitous expression of ribosomal protein genes, the ER translocation signal may be because of ceiling effects induced by the dynamic range of microarray gene expression profiling. We tested for the association using RNA sequencing data to address this concern, which has a broader dynamic range. We again observe that the ER translocation genes are enriched (100 cerebral cortex samples, AUC $=0.733, p_{\mathrm{FDR}}<10^{-9}$ ). While limited in sample coverage for donor $10021 / \mathrm{H} 0351.2002$, the RNA sequencing data validates the finding of differential expression of ER translocation genes.

\section{Estimates of cell-type proportions are disrupted in hypometabolic regions in brain 10 021/H0351.2002}

To test whether regional transcriptomic differences might be because of cell-type proportions, we performed enrichment analyses of cell-type-specific marker genes based on the differential expression results. In the five brains, microglia marker genes were expressed at low levels in the hypometabolic regions (underexpressed; $\mathrm{AUC}=0.1, p_{\mathrm{FDR}}$ $<10^{-8}$ ) while astrocyte and neuron markers were expressed at high levels (overexpressed; AUC $>0.66, p_{\mathrm{FDR}}<$ 0.05). In contrast, brain 10 021/H0351.2002 showed an opposite pattern of enrichment. Using the MGP (Mancarci et al., 2017) tool, which uses a more complex parametric method, we also observe an interaction between hypometabolic regions and brain 10021/H0351.2002, whereby estimates of microglia proportions are higher inside hypometabolic regions in brain 10021/H0351.2002 (five genes, $t=2.1, p=0.033$ ) and estimated proportions of neurons are lower (21 genes, $t=-4.0, p<0.0001)$.

\section{ER translocation gene expression is high in AD- associated microglia (DAM)}

Based on the differential expression of microglia markers in donor $10021 / \mathrm{H} 0351.2002$, we examined the

\section{continued}

blue. Genes are ordered based on hierarchical clustering using complete linkage (genes with similar expression across the mouse and human data are clustered together). Three human genes are duplicated because they have two homologous mouse genes (RPL6, RPL13, and SRP54). Human genes without homologous mouse genes are not displayed. Complete results spanning all GO groups are available as Extended Data Figure 3-1 (AD mouse model) and Extended Data Figure 3-2 (AD pathology subgroups). Data from other cell types in the AD pathology subgroups for the ER translocation genes are available in Extended Data Figure 3-3. 
ER translocation genes in microglia from an AD mouse model (Keren-Shaul et al., 2017). We tested whether the ER translocation genes increase in a stepwise pattern across the normal, intermediate, and full DAM clusters. For the 12,712 genes with data available, $6.5 \%$ monotonically increase in expression across these cell-type clusters that represent distinct states of AD-associated microglial activation. Of the 80 mouse homologs of the ER translocation genes, $75 \%$ increase in a stepwise fashion (Fig. 3, hypergeometric $\left.p<10^{-52}\right)$. Compared with all GO groups, this is the most significant enrichment (Extended Data Fig. 3-1). In this single-cell dataset, ER translocation genes are expressed in AD-associated microglia in a progressive pattern that suggests these genes increase with ADassociated microglial activation.

\section{Expression of ER translocation genes is correlated with AD pathology}

We next examined cell-type-specific transcriptomic data from postmortem human brain samples to reconnect the molecular markers with AD pathology. Specifically, using data from a single-nucleus study of the human prefrontal cortex, we next tested whether the ER translocation genes are differentially expressed across stages of AD pathology (Mathys et al., 2019). Guided by our findings in mice, we restricted our analyses to microglia. When comparing expression between no-pathology and early-pathology subgroups, we find that the ER translocation genes are enriched for higher expression in microglia from the early pathology individuals (79 genes, $\mathrm{AUC}=0.716, p<10^{-10}$ ). For the comparison between early-pathology and late-pathology subgroups, the ER translocation genes are also enriched for higher expression in the late-pathology microglia (77 genes, $A \cup C=0.627, p<0.0005$ ). Beyond these pairwise tests, we counted how many genes increase with disease progression. Broadly, for the 7319 genes with data available, the average microglial expression of $17.9 \%$ progressively increases across the pathologic groups. For the ER translocation genes, this proportion triples to $55.8 \%$ (Fig. 3, 43 of 77 genes progressively increase, hypergeometric $p<10^{-13}$ ). Compared with all GO groups, this is the second most significant group with the mostly overlapping set of cytosolic ribosome genes ranked first (Extended Data Fig. 3-2). While this relationship is strongest in microglia, astrocytes, oligodendrocytes, and their progenitor cells also have progressive increases of the ER translocation genes (proportion increased $>36 \%$, all $p<0.0002$; Extended Data Fig. 3-3). In contrast, neither inhibitory nor excitatory neurons had progressively increased ER translocation gene expression across the pathologic groups. In this single-nucleus dataset, microglial expression of the ER translocation genes is correlated with $A D$ progression.

\section{Discussion}

In this study, we projected the cerebral cortex's transcriptome onto the spatial pattern of glucose hypometabolism found in $A D$ cases. Our goal was to identify the molecular and cellular markers of this map. Of the six normal brains tested, only one demonstrated a robust spatial association between gene expression and the hypometabolism pattern. In support of this association, prior neuropathological examination of this individual found a neurofibrillary tangle. It is plausible that brain atlases seeking to assay the normal brain may contain samples from donors in the hypothetical stage of preclinical AD (Sperling et al., 2014). Our findings suggest that donor $10021 / \mathrm{H} 0351.2002$ may have been on this path.

ER translocation genes, which encode proteins of the cytosolic ribosome and target protein translation to the endoplasmic reticulum, best align with the hypometabolic pattern in brain 10021/H0351.2002. Using the transcriptomic data for this individual, we estimate a lower proportion of neurons and more microglia in hypometabolic regions. Beyond this single brain, we validate the associations between ER translocation genes and AD in microglia. Specifically, these genes have a staged expression pattern that increases across cellular and pathologic AD-associated states in human and mouse microglia. Together, these results that connect neuroimaging markers of $A D$ with single-cell signals of neuroinflammation identify ER translocation machinery as an early dysregulated process in AD.

It is striking that the ER translocation GO group was the most significantly enriched set in our analysis of the 10021/H0351.2002 donor brain and AD-associated microglia. It is known that cytosolic ribosome genes are strongly co-expressed (Lee et al., 2004). While we did not perform co-expression analysis, a change across this gene set will be easily detected with a pathway or GO analyses because of their high co-expression. This coherence is partly why it ranks above all other gene sets tested. Nonetheless, we note that a RPL34 is a topranked gene, providing a strong signal at the level of single genes. To gauge the chance of this $\mathrm{GO}$ group being top-ranked in multiple studies, we checked whether the group is multifunctional or contains commonly differentially expressed genes. We found that this group ranked average in terms of multifunctional genes, relative to other groups (ranked 6848th of 11,404 GO groups; Gillis and Pavlidis, 2011). This group was also not top-ranked in any of the 635 studies systematically examined in a broad study of differential gene expression predictability (Crow et al., 2019). More directly, the ER translocation genes are stable, with a below-average prior probability of differential expression (ER translocation genes median $=0.246$, remaining genes $=0.562$, Mann-Whitney $U$ test $p<10^{-9}$ ). Therefore, while ER translocation genes are strongly coexpressed, the prior likelihood of the ER translocation genes being differentially expressed is low.

The ribosome and protein synthesis have been previously associated with mild cognitive impairment and AD (Sajdel-Sulkowska and Marotta, 1984; Langstrom et al., 1989; Ding et al., 2005; Hernández-Ortega et al., 2016). Pathologic tau has also been shown to determine translational selectivity and co-localize with ribosomes (Meier et al., 2016; Koren et al., 2019). Beyond the ER translocation genes, we note other GO groups with functional relevance. For example, "chronic inflammatory response" 
and "cell killing" genes were enriched for overexpression in the hypometabolic regions in brain 10 021/H0351.2002. In the other direction, the genes in the homotypic fusion and protein sorting (HOPS) complex are underexpressed in hypometabolic regions in brain 10021/H0351.2002. This complex contains vacuole sorting genes and regulates autophagosome-lysosome fusion (Balderhaar and Ungermann, 2013). The top two most underexpressed gene sets in the hypometabolic regions are "ubiquitin ligase complex" and "tRNA aminoacylation." While ubiquitin ligase complex genes are underexpressed, genes encoding ubiquitin are overexpressed in the hypometabolic regions in brain 10021/H0351.2002. In summary, analysis of this single brain identifies genes that function in the protein life cycle and neuroinflammation, which are known to be disrupted in AD (Heneka et al., 2015; Gadhave et al., 2016; Cheng et al., 2018).

Intriguingly, other studies have associated the ER translocation genes with neurodegeneration. In a recent postmortem study of two cohorts, the ER translocation genes were strongly downregulated in brain samples from Parkinson's disease cases when compared with controls (Nido et al., 2020). While this contrasts our findings of upregulation, in the context of $A D$, two recent studies have also highlighted the ER translocation genes. First, an analysis of the Alzheimer's brain transcriptome found that these genes are upregulated in Caribbean-Hispanic $A D$ cases but not non-Hispanic whites (Felsky et al., 2020). The authors of this study speculate that the SRP-dependent protein targeting genes relate the gingipain hypothesis of $A D$ causation that implicates Porphyromonas gingivalis (Dominy et al., 2019). A second study supports this connection by showing that the ER translocation genes are upregulated in cortical samples with detected $P$. gingivalis sequences and are enriched for the arginine and lysine residues that the gingipain proteases cleave at (Patel et al., 2020). By performing neuroanatomical analyses, this study also discovered that the ER translocation genes are highly expressed in hypothalamus, cholinergic neurons, and the basal forebrain. This spatial signature may explain early cholinergic degeneration and sleep disruptions in $A D$. Together, our findings and these studies that implicate the same genes promote ER translocation as an underlying disease mechanism that connects the cholinergic and gingipain hypotheses of $A D$ causation.

In conclusion, the hypometabolism pattern that marks $A D$ was correlated with the expression of genes encoding ribosomal ER translocation proteins. This association was observed in the brain of a 39-year-old that contained a neurofibrillary tangle in the entorhinal cortex. In this brain, the estimated proportion of microglia was higher in the hypometabolic regions. We speculate that this individual may have been in the hypothesized preclinical stage of AD that may last decades (Sperling et al., 2011). In AD-associated microglia obtained from the cortex of 48 individuals with a broad range of $A D$ pathology, we extend these findings at the cellular level to show expression of the ER translocation genes progressively increases with $A D$ pathology. This is most pronounced in microglia from individuals with early pathology. Our transcriptomic analysis of $A D$-associated hypometabolism warrants further study of ribosomes, the protein life cycle, and neuroimmune activation in models of early $A D$.

\section{References}

Albert MS, DeKosky ST, Dickson D, Dubois B, Feldman HH, Fox NC, Gamst A, Holtzman DM, Jagust WJ, Petersen RC, Snyder PJ, Carrillo MC, Thies B, Phelps CH (2011) The diagnosis of mild cognitive impairment due to Alzheimer's disease: recommendations from the National Institute on Aging-Alzheimer's Association workgroups on diagnostic guidelines for Alzheimer's disease. Alzheimers Dement 7:270-279.

American Psychiatric Association (1994) DSM-IV. Washington, DC: American Psychiatric Association.

Ashburner M, Ball CA, Blake JA, Botstein D, Butler H, Cherry JM, Davis AP, Dolinski K, Dwight SS, Eppig JT, Harris MA, Hill DP, Issel-Tarver L, Kasarskis A, Lewis S, Matese JC, Richardson JE, Ringwald M, Rubin GM, Sherlock G (2000) Gene ontology: tool for the unification of biology. The Gene Ontology Consortium. Nat Genet 25:25-29.

Balderhaar HJK, Ungermann C (2013) CORVET and HOPS tethering complexes - coordinators of endosome and lysosome fusion. $J$ Cell Sci 126:1307-1316.

Benjamini Y, Hochberg Y (1995) Controlling the false discovery rate: a practical and powerful approach to multiple testing. J R Stat Soc Series B Stat Methodol 57:289-300.

Carlson M (2016a) GO.db: a set of annotation maps describing the entire Gene Ontology.

Carlson M (2016b) org.Hs.eg.db: genome wide annotation for human.

Chen K, Ayutyanont N, Langbaum JBS, Fleisher AS, Reschke C, Lee W, Liu X, Alexander GE, Bandy D, Caselli RJ, Reiman EM (2012) Correlations between FDG PET glucose uptake-MRI gray matter volume scores and apolipoprotein $\mathrm{E} \varepsilon 4$ gene dose in cognitively normal adults: a cross-validation study using voxel-based multimodal partial least squares. Neuroimage 60:2316-2322.

Cheng J, North BJ, Zhang T, Dai X, Tao K, Guo J, Wei W (2018) The emerging roles of protein homeostasis-governing pathways in Alzheimer's disease. Aging Cell 17:e12801.

Crow M, Lim N, Ballouz S, Pavlidis P, Gillis J (2019) Predictability of human differential gene expression. Proc Natl Acad Sci USA 116:6491-6500.

Darmanis S, Sloan SA, Zhang Y, Enge M, Caneda C, Shuer LM, Hayden Gephart MG, Barres BA, Quake SR (2015) A survey of human brain transcriptome diversity at the single cell level. Proc Natl Acad Sci USA 112:7285-7290.

Ding Q, Markesbery WR, Chen Q, Li F, Keller JN (2005) Ribosome dysfunction is an early event in Alzheimer's disease. J Neurosci 25:9171-9175.

Dominy SS, Lynch C, Ermini F, Benedyk M, Marczyk A, Konradi A, Nguyen M, Haditsch U, Raha D, Griffin C, Holsinger LJ, ArastuKapur S, Kaba S, Lee A, Ryder MI, Potempa B, Mydel P, Hellvard A, Adamowicz K, Hasturk H, et al. (2019) Porphyromonas gingivalis in Alzheimer's disease brains: evidence for disease causation and treatment with small-molecule inhibitors. Sci Adv 5:eaau3333.

Eickhoff SB, Laird AR, Grefkes C, Wang LE, Zilles K, Fox PT (2009) Coordinate-based activation likelihood estimation meta-analysis of neuroimaging data: a random-effects approach based on empirical estimates of spatial uncertainty. Hum Brain Mapp 30:29072926.

Felsky D, Sariya S, Santa-Maria I, Schneider JA, Bennett DA, Mayeux R, De Jager PL, Tosto G (2020) The Caribbean-Hispanic Alzheimer's brain transcriptome reveals ancestry-specific disease mechanisms. bioRxiv. doi: https://doi.org/10.1101/2020.05.28.122234.

Fisher RA (1925) Statistical methods for research workers. Edinburgh: Oliver and Boyd. 
French L, Paus T (2015) A FreeSurfer view of the cortical transcriptome generated from the Allen Human Brain Atlas. Front Neurosci 9:323.

Friedland RP, Budinger TF, Ganz E, Yano Y, Mathis CA, Koss B, Ober BA, Huesman RH, Derenzo SE (1983) Regional cerebral metabolic alterations in dementia of the Alzheimer type: positron emission tomography with [18F]fluorodeoxyglucose. J Comput Assist Tomogr 7:590-598.

Gadhave K, Bolshette N, Ahire A, Pardeshi R, Thakur K, Trandafir C, Istrate A, Ahmed S, Lahkar M, Muresanu DF, Balea M (2016) The ubiquitin proteasomal system: a potential target for the management of Alzheimer's disease. J Cell Mol Med 20:1392-1407.

Gillis J, Pavlidis P (2011) The impact of multifunctional genes on "guilt by association" analysis. PLoS One 6:e17258.

Grothe MJ, Sepulcre J, Gonzalez-Escamilla G, Jelistratova I, Schöll M, Hansson O, Teipel SJ, Alzheimer's Disease Neuroimaging Initiative. (2018) Molecular properties underlying regional vulnerability to Alzheimer's disease pathology. Brain 141:2755-2771.

Guillozet AL, Weintraub S, Mash DC, Mesulam MM (2003) Neurofibrillary tangles, amyloid, and memory in aging and mild cognitive impairment. Arch Neurol 60:729-736.

Hawrylycz MJ, Lein ES, Guillozet-Bongaarts AL, Shen EH, Ng L, Miller JA, van de Lagemaat LN, Smith KA, Ebbert A, Riley ZL, Abajian C, Beckmann CF, Bernard A, Bertagnolli D, Boe AF, Cartagena PM, Chakravarty MM, Chapin M, Chong J, Dalley RA, et al. (2012) An anatomically comprehensive atlas of the adult human brain transcriptome. Nature 489:391-399.

Hawrylycz M, Miller JA, Menon V, Feng D, Dolbeare T, GuillozetBongaarts AL, Jegga AG, Aronow BJ, Lee CK, Bernard A, Glasser MF, Dierker DL, Menche J, Szafer A, Collman F, Grange P, Berman KA, Mihalas S, Yao Z, Stewart L, et al. (2015) Canonical genetic signatures of the adult human brain. Nat Neurosci 18:1832-1844.

Heneka MT, Carson MJ, Khoury JE, Landreth GE, Brosseron F, Feinstein DL, Jacobs AH, Wyss-Coray T, Vitorica J, Ransohoff RM, Herrup K, Frautschy SA, Finsen B, Brown GC, Verkhratsky A, Yamanaka K, Koistinaho J, Latz E, Halle A, Petzold GC, et al. (2015) Neuroinflammation in Alzheimer's disease. Lancet Neurol 14:388-405.

Hernández-Ortega K, Garcia-Esparcia P, Gil L, Lucas JJ, Ferrer I (2016) Altered machinery of protein synthesis in Alzheimer's: from the nucleolus to the ribosome. Brain Pathol 26:593-605.

Keren-Shaul H, Spinrad A, Weiner A, Matcovitch-Natan O, DvirSzternfeld R, Ulland TK, David E, Baruch K, Lara-Astaiso D, Toth B, Itzkovitz S, Colonna M, Schwartz M, Amit I (2017) A unique microglia type associated with restricting development of Alzheimer's disease. Cell 169:1276-1290.e17.

Koren SA, Hamm MJ, Meier SE, Weiss BE, Nation GK, Chishti EA, Arango JP, Chen J, Zhu H, Blalock EM, Abisambra JF (2019) Tau drives translational selectivity by interacting with ribosomal proteins. Acta Neuropathol 137:571-583.

Landau SM, Harvey D, Madison CM, Koeppe RA, Reiman EM, Foster NL, Weiner MW, Jagust WJ; Alzheimer's Disease Neuroimaging Initiative (2011) Associations between cognitive, functional, and FDG-PET measures of decline in $A D$ and $\mathrm{MCl}$. Neurobiol Aging 32:1207-1218.

Langbaum JBS, Chen K, Caselli RJ, Lee W, Reschke C, Bandy D, Alexander GE, Burns CM, Kaszniak AW, Reeder SA, Corneveaux JJ, Allen AN, Pruzin J, Huentelman MJ, Fleisher AS, Reiman EM (2010) Hypometabolism in Alzheimer-affected brain regions in cognitively healthy Latino individuals carrying the apolipoprotein $\mathrm{E}$ epsilon4 allele. Arch Neurol 67:462-468.

Langstrom NS, Anderson JP, Lindroos HG, Winblad B, Wallace WC (1989) Alzheimer's disease-associated reduction of polysomal mRNA translation. Brain Res Mol Brain Res 5:259-269.

Lee HK, Hsu AK, Sajdak J, Qin J, Pavlidis P (2004) Coexpression analysis of human genes across many microarray data sets. Genome Res 14:1085-1094.

Mancarci BO, Toker L, Tripathy SJ, Li B, Rocco B, Sibille E, Pavlidis P (2017) Cross-laboratory analysis of brain cell type transcriptomes with applications to interpretation of bulk tissue data. eNeuro 4:ENEURO.0212-17.2017.

Masters CL, Bateman R, Blennow K, Rowe CC, Sperling RA, Cummings JL (2015) Alzheimer's disease. Nat Rev Dis Primers 1:15056.

Mathys H, Davila-Velderrain J, Peng Z, Gao F, Mohammadi S, Young JZ, Menon M, He L, Abdurrob F, Jiang X, Martorell AJ, Ransohoff RM, Hafler BP, Bennett DA, Kellis M, Tsai LH (2019) Single-cell transcriptomic analysis of Alzheimer's disease. Nature 570:332-337.

McKhann G, Drachman D, Folstein M, Katzman R, Price D, Stadlan EM (1984) Clinical diagnosis of Alzheimer's disease: report of the NINCDS-ADRDA Work Group under the auspices of Department of Health and Human Services Task Force on Alzheimer's Disease. Neurology 34:939-944.

McKhann GM, Knopman DS, Chertkow H, Hyman BT, Jack CR Jr, Kawas CH, Klunk WE, Koroshetz WJ, Manly JJ, Mayeux R, Mohs RC, Morris JC, Rossor MN, Scheltens P, Carrillo MC, Thies B, Weintraub S, Phelps CH (2011) The diagnosis of dementia due to Alzheimer's disease: recommendations from the National Institute on Aging-Alzheimer's Association workgroups on diagnostic guidelines for Alzheimer's disease. Alzheimers Dement 7:263-269.

Meier S, Bell M, Lyons DN, Rodriguez-Rivera J, Ingram A, Fontaine $\mathrm{SN}$, Mechas E, Chen J, Wolozin B, LeVine H 3rd, Zhu H, Abisambra JF (2016) Pathological tau promotes neuronal damage by impairing ribosomal function and decreasing protein synthesis. J Neurosci 36:1001-1007.

Miller JA, Menon V, Goldy J, Kaykas A, Lee CK, Smith KA, Shen EH, Phillips JW, Lein ES, Hawrylycz MJ (2014) Improving reliability and absolute quantification of human brain microarray data by filtering and scaling probes using RNA-Seq. BMC Genomics 15:154.

Mosconi L, Sorbi S, de Leon MJ, Li Y, Nacmias B, Myoung PS, Tsui W, Ginestroni A, Bessi V, Fayyazz M, Caffarra P, Pupi A (2006) Hypometabolism exceeds atrophy in presymptomatic early-onset familial Alzheimer's disease. J Nucl Med 47:1778-1786.

Mosconi L, Tsui WH, Herholz K, Pupi A, Drzezga A, Lucignani G, Reiman EM, Holthoff V, Kalbe E, Sorbi S, Diehl-Schmid J, Perneczky R, Clerici F, Caselli R, Beuthien-Baumann B, Kurz A, Minoshima S, de Leon MJ (2008) Multicenter standardized 18FFDG PET diagnosis of mild cognitive impairment, Alzheimer's disease, and other dementias. J Nucl Med 49:390-398.

Nido GS, Dick F, Toker L, Petersen K, Alves G, Tysnes O-B, Jonassen I, Haugarvoll K, Tzoulis C (2020) Common gene expression signatures in Parkinson's disease are driven by changes in cell composition. Acta Neuropathol Commun 8:55.

Oddo S (2008) The ubiquitin-proteasome system in Alzheimer's disease. J Cell Mol Med 12:363-373.

Patel S, Howard D, French L (2020) Susceptibility to gingipains and transcriptomic response to $P$. gingivalis highlights the ribosome, hypothalamus, and cholinergic neurons. bioRxiv. doi: https://doi. org/10.1101/2020.08.09.243402.

Qiu C, Kivipelto M, von Strauss E (2009) Epidemiology of Alzheimer's disease: occurrence, determinants, and strategies toward intervention. Dialogues Clin Neurosci 11:111-128.

Reiman EM, Chen K, Alexander GE, Caselli RJ, Bandy D, Osborne D, Saunders AM, Hardy J (2004) Functional brain abnormalities in young adults at genetic risk for late-onset Alzheimer's dementia. Proc Natl Acad Sci USA 101:284-289.

Ritchie J, Pantazatos SP, French L (2018) Transcriptomic characterization of MRI contrast with focus on the T1-w/T2-w ratio in the cerebral cortex. Neuroimage 174:504-517.

Sajdel-Sulkowska EM, Marotta CA (1984) Alzheimer's disease brain: alterations in RNA levels and in a ribonuclease-inhibitor complex. Science 225:947-949.

Sperling RA, Aisen PS, Beckett LA, Bennett DA, Craft S, Fagan AM, Iwatsubo T, Jack CR, Kaye J, Montine TJ, Park DC, Reiman EM, Rowe CC, Siemers E, Stern Y, Yaffe K, Carrillo MC, Thies B, Morrison-Bogorad M, Wagster MV, et al. (2011) Toward defining the preclinical stages of Alzheimer's disease: recommendations from 
the National Institute on Aging-Alzheimer's Association workgroups on diagnostic guidelines for Alzheimer's disease. Alzheimers Dement 7:280-292.

Sperling R, Mormino E, Johnson K (2014) The evolution of preclinical Alzheimer's disease: implications for prevention trials. Neuron 84:608-622.
Supek F, Bošnjak M, Škunca N, Šmuc T (2011) REVIGO summarizes and visualizes long lists of gene ontology terms. PLoS One 6: e21800.

Turkeltaub PE, Eden GF, Jones KM, Zeffiro TA (2002) Meta-analysis of the functional neuroanatomy of single-word reading: method and validation. Neuroimage 16:765-780. 